\title{
Qualidade de Vida em Idosos portadores de Hipertensão Arterial e Diabetes Mellitus
}

\author{
Danilo Pereira de Souza ${ }^{1}$, Thamy Silveira Melo², Luciana Araújo dos Reis ${ }^{3}$, Pollyanna Viana Lima ${ }^{4}$
}

\begin{abstract}
Resumo: O presente estudo tem com objetivo avaliar a qualidade de vida de idosos. Trata-se de um estudo transversal, descritivo-quantitativo, com amostra constituída por 50 idosos cadastrados em uma Estratégia de Saúde da Família do interior da Bahia. Os instrumentos utilizados para a coleta dos dados foram o Mini-Exame do Estado Mental, o questionário sociodemográfico e econômico e o WHOQOL-OLD e WHOQOL- BREF. Para a análise dos dados foi utilizado o programa estatístico SPSS $®$, versão 20.0. Na avaliação da qualidade de vida observou-se que os participantes obtiveram as piores pontuações nos domínios psicológico (51,30 pontos) e ambiente (59,98 pontos) e as facetas mais comprometidas foram Autonomia (44,96 pontos) e Atividades passadas, presentes e futuras (48,80 pontos). Conclui-se que o processo de envelhecimento dos indivíduos participantes do estudo está relacionado a fatores que refletem em impactos negativos na qualidade de vida.
\end{abstract}

Palavras-chave: Idoso, Qualidade de Vida, Hipertensão Arterial, Diabetes Mellitus.

\section{Quality of Life in Elderly with Arterial Hypertension and Diabetes Mellitus}

\begin{abstract}
The present study is to evaluate the quality of life of seniors. This is a cross-sectional, descriptive and quantitative study, with sample of 50 elderly enrolled in a health strategy for the interior of Bahia Family. The instruments used for data collection were the Mini-Mental State Examination, the socio-demographic and economic questionnaire and WHOQOL-OLD and WHOQOL-BREF. For data analysis was used the statistical program SPSS, version 20.0. In evaluating the quality of life was observed that the participants obtained the lowest scores in the psychological (51.30 points) and environment (59.98 points) and the most committed aspects were Autonomy (44.96 points) and past activities, present and future (48.80 points). We conclude that the aging process of the study participants is related to factors that reflect negative impacts on quality of life.
\end{abstract}

Keywords: Old man, Quality of Life, Arterial hypertension, Diabetes mellitus.

\section{Introdução}

Os indicadores de envelhecimento da população, segundo dados estatísticos, mostram que houve um aumento acentuado do número de idosos nos últimos milênios. Conforme os estudos prelecionados por uma pesquisa em 2013, estimativas tem apontado que até 2025 o número de idosos será superior a 30 milhões (DAWALIBI et al, 2013).

\footnotetext{
${ }^{1}$ Faculdade Independente do Nordeste-FAINOR; danilosouzars@ gmail.com

${ }^{2}$ Enfermeira da Estratégia de Saúde da Família. Especialista em Enfermagem do trabalho. Faculdade Independente do Nordeste-FAINOR. tlifemelo@gmail.com

${ }^{3}$ Doutora em Ciências da Saúde pela UFRN. Professora Titular do Programa de Pós - Graduação Stricto Sensu em Memória: Linguagem e Sociedade e do Curso de Fisioterapia da UESB. Professora da Faculdade Indenpendente do Nordeste - FAINOR. lucianauesb@yahoo.com.br

${ }^{4}$ Doutoranda em Memória: Linguagem e Sociedade da Universidade Estadual do Sudoeste da Bahia - UESB. Docente do curso de Enfermagem da Faculdade Independente do Nordeste - FAINOR. polly_vl@yahoo.com.br
} 
Concomitante a esse crescimento, alguns autores salientam que essa velhice vem acompanhada por diversas modificações no organismo humano, incluindo as doenças crônicas, que englobam uma série de patologias não-transmissíveis, tais como: Hipertensão Arterial Sistêmica (HAS), Diabetes Mellitus (DM), doenças osteomusculares, dentre outras (MACHADO; CAVALIÉRE, 2012).

De acordo com estudos realizados, as Doenças Crônicas Não Transmissíveis (DCNT) configuram-se como um grave problema de saúde pública, não somente pelos elevados custos para o Sistema Único de Saúde, como também pelas consequências que essas patologias acarretam a vida do idoso, implicando em alguns casos em óbitos, em outros perda da capacidade funcional e, por conseguinte, perda da qualidade de vida (MALTA et al, 2014).

Nesse sentido, é possível afirmar que a Qualidade de Vida (QV) é um excelente preditor para o envelhecimento. Segundo a Organização Mundial de Saúde qualidade de vida é "a percepção do indivíduo de sua posição na vida, no contexto de sua cultura e dos sistemas de valores da sociedade em que vive e em relação aos seus objetivos, expectativas, padrões e preocupações" (SANTOS, 2015, p. 06). Pelo exposto pela OMS, observa-se, pois, que a saúde engloba não somete o aspecto físico do indivíduo, como também o seu estado psicológico, o nível de independência, capacidade funcional, dentre outros aspectos (GUEDES et al, 2013).

A motivação maior que levou à escolha do tema foi o desejo de colaborar com o conhecimento de uma questão de grande relevância para a sociedade brasileira: a qualidade de vida em idosos. Do ponto de vista acadêmico, o assunto é de amplo interesse para o contexto social, pois o tema lida diretamente com uma população que tem crescido muito no Brasil nas últimas décadas e que é muito acometida com doenças crônico-degenerativas.

Além disso, o estudo realizado pode contribuir para o aperfeiçoamento da temática aqui tratada, uma vez que possibilitará conhecer como está a qualidade de vida de idosos cadastrados em uma Estratégia de Saúde da Família e os impactos dessas patologias na qualidade de vida dos idosos.

Diante dos aspectos mencionados, a insuficiência de informações e a importância de pesquisas envolvendo idosos, justifica-se a relevância deste estudo. $\mathrm{O}$ número de pesquisas relacionadas ao envelhecimento vem aumentando nos últimos anos. Todavia, poucos são as pesquisas que tratam do impacto das doenças crônicas degenerativas na qualidade de vida de idosos, especialmente quando se trata de pesquisas em cidades pequenas do interior da Bahia. 
Id on Line Revista Multidisciplinar e de Psicoloqia

Id on Line Multidisciplinary Journal and Psycology

Assim, considerando que as questões que envolvem o idoso, a qualidade de vida e sua relação com as doenças crônico-degenerativas são de extrema complexidade e requer estudos cada vez mais abrangentes para dar conta do mesmo, surgiu a seguinte questão problema: Como está a qualidade de vida dos idosos portadores de hipertensão arterial sistêmica e diabetes mellitus cadastrados em uma ESF do interior da Bahia?

Para tanto, têm-se como objetivo avaliar a qualidade de vida de idosos portadores de HAS e DM cadastrados em uma ESF do interior da Bahia.

\section{Metodologia}

Trata-se de um estudo transversal do tipo descritivo, com abordagem quantitativa. Os estudos descritivos utilizam técnicas padronizadas de coleta de dados, tais como questionário e a observação. A pesquisa quantitativa tem seus resultados quantificados, além disso, centrase na objetividade, pois apela à linguagem matemática para descrever as causas de um fenômeno, as relações entre variáveis (GIL, 2010).

A presente pesquisa foi realizada em uma ESF de um município do interior da Bahia. Os participantes do estudo foram idosos de 60 anos ou mais de ambos os sexos, hipertensos e diabéticos, cadastrados no programa HIPERDIA da ESF do município onde ocorreu o estudo, que apresentavam condições mentais para responder aos questionários (avaliados por meio do Mini Exame do Estado Mental - MEEM) e que aceitaram participar do estudo. A seleção da amostra foi realizada por meio de amostragem aleatória e foi considerado aqueles idosos que se apresentarem no dia da reunião do HIPERDIA, bem como no dia da busca do medicamento, sendo a amostra representada por 50 idosos.

A coleta de dados ocorreu na própria ESF, em uma sala reservada, nos dias das reuniões do HIPERDIA e do recebimento de medicação. Para a execução dos objetivos propostos ao longo do trabalho, foram aplicados quatro instrumentos de pesquisa: o MEEM; um questionário Social, demográfico e Econômico e dois questionários de Qualidade de Vida.

Para avaliação do estado mental foi aplicado o Mini-exame do Estado Mental de Pfeiffer, composto por dez perguntas, o qual avalia quatro parâmetros: memória a curto e longo prazo; orientação; informações do cotidiano; e capacidade de cálculo (FOLSTEIN et al, 
1975). A pontuação seguiu o seguinte critério: 30 a 26 pontos (funções cognitivas preservadas); 26 a 24 pontos (alteração não sugestiva de déficit) e 23 pontos ou menos (sugestivo de déficit cognitivo). Esse Mini-exame do estado mental possibilitará a exclusão dos idosos que não apresentavam cognição para responder às perguntas da entrevista.

A fim de caracterizar o perfil sociodemográfico e econômico da população em estudo será aplicado o questionário sociodemográfico e econômico construído pelos próprios pesquisadores. Os dados serão relacionados à: idade, sexo, cor da pele, estado civil, renda, moradia, escolaridade, religião e ocupação.

Os dados referentes à qualidade de vida foram coletados por meio de dois instrumentos: O WHOQOL-OLD e o WHOQOL-BREF. O WHOQOL-OLD é um instrumento de fácil aplicação, validado e adaptado a população brasileira, em específico para a população idosa. O Módulo WHOQOL-OLD é constituído de 24 perguntas e suas respostas seguem uma escala de Likert (de 1 a 5) atribuídos a seis facetas: funcionamento sensorial, autonomia, atividades passadas, presentes e futuras, participação social, morte e morrer e intimidade) e mede a percepção dos indivíduos acerca dos impactos que as doenças causam em suas vidas. Cada uma das facetas possui 4 perguntas; podendo as respostas oscilar de 4 a 20. Escores altos representam uma alta qualidade de vida, escores baixos representam uma baixa qualidade de vida (OMS, 2012).

O WHOQOL-BREF também é um instrumento de avaliação de qualidade de vida, elaborado em 1988 por um grupo multicêntrico interessados em estudos sobre qualidade de vida da Organização Mundial da Saúde (OMS, 2012).

Ele é derivado do WHOQOL-100, assim denominado por conter 100 questões que agrupadas formam seis dimensões ou domínios: físico, psicológico, nível de independência, relações pessoais, ambiente, espiritualidade. A versão abreviada WHOQOL-BREF é composta por 26 questões que abrange quatro domínios: físico, psicológico, relações pessoais e meio-ambiente. $\mathrm{O}$ escore para cada domínio pode ser transformado em uma escala que varia de 0-100, sendo zero o pior e 100 o melhor resultado Esse instrumento é um é um pouco diferente do WHOQOL-OLD. Neste instrumento terá que aparecer o resultado somente em média (1 a 5) por domínio e por faceta (OMS, 2012).

Após a coleta dos dados, foi realizada análise descritiva das variáveis de estudo, por meio da distribuição de frequência absoluta e relativa para as variáveis categóricas e medidas 
Id on Line Revista Multidisciplinar e de Psicoloqia

Id on Line Multidisciplinary Journal and Psycology

de tendência central (média, mediana e moda) e dispersão (desvio-padrão e amplitude), para as variáveis contínuas. Para testar a diferença entre duas médias foi utilizado o teste $\mathrm{t}$ de Student e, entre três ou mais médias, o teste ANOVA. As diferenças estatísticas entre proporções serão avaliadas usando-se os testes de qui-quadrado de Pearson ou exato de Fisher, com um nível de significância de $5 \%(\mathrm{p}<0,05)$.

Em todas as análises foram fixados valores de confiança de 5\% $(\alpha=0,05)$. Para a elaboração do banco de dados foi utilizado o aplicativo EPIDATA e, para a análise dos dados, o programa estatístico SPSS ${ }^{\circ}$, versão 20.0. Das informações obtidas a partir dos questionários e da análise estatística já mencionada, foi feita uma interpretação, dialogando com a literatura que discute a temática, com o intuito de responder às questões da pesquisa e assim alcançar o objetivo do estudo.

Todos os aspectos éticos e legais da Resolução 466/12 do Ministério da Saúde foram seguidos rigorosamente. O projeto foi submetido e aprovado pelo Comitê de Ética em Pesquisa da Faculdade Independente do Nordeste (FAINOR), com Parecer $n^{\circ}$ 1.687.752. Todos os idosos participantes do estudo assinaram um Termo de Consentimento Livre e Esclarecido (TCLE), após a explicação dos objetivos do estudo e os procedimentos da coleta das informações.

\section{Resultados e Discussões}

Quanto as características sociodemográficas, econômicas e de saúde constatou-se no presente estudo que a média das idades dos idosos foi de 70 anos, houve uma maior frequência de idosos do sexo feminino (66,0\%), casado/União Estável (66,0\%), com ocupação referente a trabalho rural $(64,0 \%)$, que não trabalha atualmente $(54,0 \%)$, com renda individual de 1 salário mínimo $(92,0 \%)$, renda familiar total de 1 salário mínimo $(82,0 \%)$; portador de HAS (64\%), portador de DM (14\%) e portador de HAS e DM (22\%).

Tanto no WHOQOL-BREF, como no WHOQOL-OLD não existem limiares capazes de determinar um escore abaixo ou acima no qual se possa avaliar a qualidade de vida como boa ou ruim (SILVA et al, 2012). Portanto, para avaliação dos escores encontrados no 
Id on Line Revista Multidisciplinar e de Psicoloqia

Id on Line Multidisciplinary Journal and Psycology

presente estudo, os valores mais baixos foram caracterizados como: qualidade de vida insatisfatória, ou, qualidade de vida mais comprometida.

Em relação aos domínios apresentados na Tabela 1, referente ao questionário de QV WHOQOL-BREF, observou-se que os participantes obtiveram a melhor pontuação no domínio Relações Sociais (79,92 pontos) e as piores pontuações nos domínios psicológico (51,30 pontos) e ambiente (59,98 pontos).

Tabela 1. Distribuição dos domínios do Whoqol-bref. Vitória da Conquista/BA, 2016.

\begin{tabular}{lcccc}
\hline Domínios & N & Média & $\begin{array}{c}\text { Desvio } \\
\text { Padrão }\end{array}$ & IC \\
\hline Domínio Físico & 50 & 63,39 & 14,61 & 4,63 \\
Domínio Psicológico & 50 & 51,30 & 10,94 & 4,63 \\
Domínio Relações Sociais & 50 & 79,92 & 14,43 & 8,42 \\
Domínio Ambiente & 50 & 59,98 & 8,14 & 5,4 \\
\hline
\end{tabular}

Fonte: Dados da pesquisa

Discutir QV é uma tarefa difícil, uma vez que abrange diversos aspectos (físico, mental, social), na vida de um indivíduo seja ele portador de alguma patologia crônica ou não (SILVA et al, 2012). Em outras palavras, a QV é “a percepção do indivíduo em relação a sua vida num contexto cultural dentro do sistema em que ele está inserido e em relação a seus objetivos, suas expectativas, seus padrões e suas preocupações" (WHO, 1995, p. 20).

Em indivíduos adultos saudáveis, a qualidade de vida é um estado ou condição benéfica de vida, em que aspectos como: bem estar físico, mental, emocional e social, são avaliados e uma vez que estes não estejam adequados para a realidade do indivíduo, podem interferir diretamente no seu bem estar (SILVA et al, 2012). Especificamente em relação a idosos, a QV pode ser caracterizada como um conceito complexo e multidimensional que envolve aspectos relacionados à saúde, aspectos sociais e estruturais (PRAZERES; FIGUEIREDO, 2014).

O domínio de Relações Sociais envolve as relações pessoais, o suporte (apoio) social e a atividade sexual. Este domínio foi o que apresentou a melhor pontuação $(79,92)$, ou seja, o que mais contribuiu na qualidade de vida dos idosos, indicando que aqueles que possuem 
Id on Line Revista Multidisciplinar e de Psicoloqia

Id on Line Multidisciplinary Journal and Psycology

bons relacionamentos nos meios sociais e não se isolam, apresentam maior satisfação pessoal, o que sem dúvida minimiza a ocorrência de possíveis sentimentos de tristeza.

Resultado que se assemelha ao realizado por Dahmer et al (2015) e de Stival et al (2014), também com idosos. Vale ressaltar que as relações sociais para idosos são extremamente significativas, possibilitam um melhor convívio social, permite ao idoso que continue ativo e participativo na sociedade. O que consequentemente melhora a autoestima e reduz sentimentos de isolamento e tristeza.

Os domínios psicológico e ambiental apresentaram valores mais baixos em relação aos demais domínios. Estes baixos valores indicam um possível comprometimento na QV dos idosos, no que diz respeito a sentimentos positivos e negativos, memória e concentração, auto-estima, imagem corporal, segurança física e proteção, recursos financeiros etc. (BECKERT et al, 2012; NORONHA et al, 2016).

Além dos fatores supracitados, existem ainda as "perdas", tais como o surgimento das doenças crônicas-degenerativas, viuvez, a morte dos amigos e parentes, ausência de papéis sociais valorizados, isolamento crescente, que refletem diretamente em uma qualidade de vida baixa. Além disso, uma série de estudos tem mostrado o quanto as DCNT, como a HAS e a DM, interferem em uma boa qualidade de vida para idosos (SANTOS et al, 2013; MALTA et al, 2014).

Um estudo de base populacional, realizado com 1.467 indivíduos coreanos avaliou o impacto da hipertensão arterial, diabetes mellitus e doenças cardiovasculares na QV dos idosos. Os resultados mostraram impactos extremamente negativos, sendo que idosos com mais de uma comorbidade associada tinham qualidade de vida pior em relação àqueles que tinham apenas uma morbidade. Todos os aspectos físicos avaliados como: mobilidade, dor, desconforto, auto cuidado, apresentaram alterações negativas relacionadas à associação das comorbidades. Os piores impactos foram observados nas condições psicológicas dos indivíduos, que apresentam elevadas taxas de ansiedade e depressão devido a associação das doenças (CHIN et al, 2014).

Em outro estudo, também de base populacional, realizado no ano de 2014 em Portugal, com objetivo de avaliar apenas os impactos do DM do tipo 2 na QV dos idosos de uma Unidade Básica de Saúde, mostraram que os domínios mais afetados pela patologia foram: saúde mental e bem estar (PRAZERES; FIGUEIREDO, 2014). Os resultados dos 
Id on Line Revista Multidisciplinar e de Psicoloqia

Id on Line Multidisciplinary Journal and Psycology

estudos supracitados são semelhantes ao encontrados no presente estudo, uma vez que foi identificado a qualidade de vida mais comprometida no domínio psicológico (51,30 pontos).

Ficou evidenciado também que a QV encontra-se comprometida no domínio ambiental, o que deixa claro que as boas condições de moradia, segurança física, recursos financeiros, transporte, aquisição de alguns bens de consumo, dentre outros podem estar associados para melhoria da qualidade de vida. Outro estudo também evidenciou resultados semelhantes, onde o domínio ambiental com baixo escore também interfere em uma boa qualidade de vida (SILVA et al, 2012).

Diante disso, fica claramente evidenciado que baixos escores nos domínios psicológicos e ambiental tem repercussão negativa em relação a HAS e a Diabetes Mellitus.

$\mathrm{Na}$ avaliação da qualidade de vida por meio do WHOQOL-OLD as facetas mais comprometidas foram Autonomia (44,96 pontos) e Atividades passadas, presentes e futuras ( 48,80 pontos) e as melhores médias foram encontradas na faceta Intimidade ( 62,80 pontos) e no Funcionamento Sensório Motor (61,56 pontos), segundo dados da Tabela 2.

Tabela 2. Distribuição dos domínios do Whoqol-old. Vitória da Conquista/BA, 2016.

\begin{tabular}{lcccc}
\hline Facetas & N & Média & padrão & IC \\
\hline Funcionamento do sensório & 50 & 61,56 & 2,14 & 13,90 \\
Autonomia & 50 & 44,96 & 3,67 & 32,65 \\
Atividades passadas, presentes e & & & & \\
futuras & 50 & 48,80 & 2,95 & 24,18 \\
Participação social & 50 & 51,16 & 3,16 & 24,73 \\
Morte e morrer & 50 & 52,48 & 3,37 & 25,70 \\
Intimidade & 50 & 62,80 & 2,71 & 17,28 \\
\hline
\end{tabular}

Fonte: Dados da pesquisa

As diversas mudanças fisiológicas que ocorrem no processo de envelhecimento são capazes de causar em alguns idosos o declínio na capacidade funcional em médio e longo prazo, tornando-os alvos para fragilidade e maior dependência de cuidados, refletindo na diminuição da autonomia do mesmo (FARIAS; SANTOS, 2012). 


\begin{abstract}
Autonomia sugere tomada de decisão deliberada ou mais livre, preservação da integridade e individualidade, baseada em aspirações, valores, crenças e objetivos particulares de cada ser. A hierarquização do cuidado e o empoderamento dos saberes em torno do processo saúde e doença, acrescidos às deliberações dos códigos de ética, cerceiam a tomada de decisão do idoso, favorecendo a sua passividade (CARRETA et al, 2011, p. 960).
\end{abstract}

Muitos idosos veem sua qualidade de vida predestinada a deteriorar-se com o passar dos anos, pois sempre atribuem a velhice com perca da capacidade de autonomia e perca da independência. Muitos temem a velhice pela possibilidade de se tornarem dependentes dos filhos ou de cuidadores, por algum tipo de doença ou por não conseguir mais executar as atividades cotidianas (FREITAS et al, 2010). Um estudo publicado em 2014, salienta que a falta de adaptação dos idosos em relação aos novos papeis sociais, os levam a desenvolver estes pensamentos e atitudes negativas de perca da autonomia e independência (BIOLCHI et al, 2014).

O profissional de saúde da atenção básica e demais níveis de atenção à saúde devem desmitificar essa visão equivocada que os idosos têm, e devem reafirmar que a autonomia e autocuidado podem ser influenciadas pelo bom estado físico, pois se o idoso possui um bom estado físico a sua autonomia é preservada mais facilmente quando comparado a um idoso que tem envelhecimento não saudável e mau estado físico. Além disso, a manutenção da autonomia leva o idoso a sentir-se mais seguro para viver de forma independente (BECKERT et al, 2012).

Atualmente "o envelhecimento ativo é uma ideologia [...] capaz de permitir [...] que as pessoas percebam o seu potencial, participem da sociedade de acordo com as suas especificidades, além de lhes propiciar proteção, segurança e cuidados adequados, quando necessários.” (FARIAS; SANTOS, 2012, p. 168).

Um estudo brasileiro de base populacional, realizado com 841 idosos e publicado em 2016, mostra o quanto a prática de atividade física é relevante para a saúde dos idosos. Somente $20 \%$ da população estudada praticava a ideologia do envelhecimento ativo, e nesta parcela, a qualidade de vida e capacidade física apresentavam-se elevadas e melhores em relação aos demais, ficando mais uma vez evidenciado a importância da prática de atividades físicas durante a vida e o processo de envelhecimento (NORONHA et al, 2016).

Outro estudo, realizado em 2012 que também mensurou a qualidade de vida em idosos que praticavam o envelhecimento ativo, evidenciou que frequentar grupos de convivência 
Id on Line Revista Multidisciplinar e de Psicoloqia

Id on Line Multidisciplinary Journal and Psycology

para idosos melhora a qualidade de vida, principalmente nos aspectos emocionais e físicos. Os escores dos idosos do estudo foram $(69,82)$ no domínio emocional e $(73,82)$ nos aspectos físicos (LEITE et al, 2012).

$\mathrm{Na}$ faceta atividades passadas, presentes e futuras, os baixos escores identificaram comprometimento na qualidade de vida dos idosos. Nesta faceta, itens como satisfação dos idosos com as conquistas no decorrer da vida e as perspectivas futuras foram avaliadas. "As expectativas das pessoas variam de acordo com o seu nível sócio-econômico-cultural, o acesso aos serviços de saúde, os anseios da família e da comunidade, o nível de dependência, custo e financiamento do tratamento, entre outros" (TAVARES et al, 2011).

Um estudo realizado com 542 idosos hipertensos e publicado no ano de 2015, também mostra resultados semelhantes para esta faceta e atribui maior impacto ao grande percentual de idosos com diversas comorbidades associadas, que podem levar o individuo a diminuir suas perspectivas em relação ao futuro (TAVARES et al, 2015).

Diante do exposto, fica evidente que a autonomia e as atividades cotidianas dos indivíduos, especialmente os portadores de DCNT, como a HAS e a DM, podem ser afetadas durante o processo de envelhecimento, porém deve-se estimular o idoso a adotar a ideologia do envelhecimento ativo e autônomo.

As facetas de maiores escores foram: Intimidade (62,80 pontos) e no Funcionamento Sensório Motor (61,56 pontos). Na faceta intimidade é avaliada a capacidade que o idoso tem em se relacionar com outras pessoas. Um estudo que encontrou resultados semelhantes atribui este dado para o maior número de idosos que vivem com a família ou que tem cônjuge (SANTOS et al, 2013).

Com relação ao Funcionamento Sensório Motor, os altos escores indicam que os idosos conseguem executar suas atividades diárias sem grandes prejuízos. O estudo realizado por Braz; Zaia; Bittar, demonstrou que os bons escores nesta faceta foram de idosos que freqüentam grupos de convivência ou praticavam atividade física, o que reforça mais uma vez o impacto positivo da ideologia do envelhecimento ativo como citado anteriormente (BRAZ et al, 2015). 
Id on Line Revista Multidisciplinar e de Psicologia

Id on Line Multidisciplinary Journal and Psycology

\section{Considerações Finais}

Os resultados obtidos com o presente estudo, que avaliou a qualidade de vida de idosos portadores de HAS e DM cadastrados em uma ESF do interior da Bahia, demonstraram que a QV de idosos portadores de HAS e DM é comprometida, especialmente nos domínios psicológico e ambiental e nas facetas autonomia, atividades presentes, passadas e futuras. O que sugere que o processo de envelhecimento dos indivíduos participantes do estudo está arraigado de fatores que refletem em impactos negativos na $\mathrm{QV}$, dentre os fatores avaliados destaca-se a própria presença de HAS e DM, ou associação das duas morbidades.

As limitações do estudo referem-se ao quantitativo de participantes uma vez que os resultados desta população não representam uma realidade de um grupo populacional. Todavia, acredita-se que pela exiguidade de estudos sobre a temática, com idosos de zona rural, esta pesquisa poderá incentivar o desenvolvimento de outros estudos que possam dar conta de uma população mais abrangente.

Mas é preciso salientar que mesmo sendo um estudo que se refere a uma população específica é de extrema pertinência para pensar práticas preventivas para a população idosa portadora de HAS e DM, haja vista a importância atual das temáticas mobilizadas neste estudo, especialmente pelo fato de proporcionar, a partir de informações científicas, o planejamento e a implementação de ações de responsabilidade das esferas governamentais, no desenvolvimento de políticas públicas, voltadas à melhoria da qualidade de vida dos indivíduos.

\section{Referências}

BECKERT, M.; IRIGARAY, T. Q.; TRENTINI, C. M. Quality of life, cognition and performance of executive functions in the elderly. Estudos de Psicologia, v. 29, n. 2, p. 155$162,2012$.

BIOLCHI, C. S. da.; PORTELLA, M. R.; COLUSSI, E. L. Vida e velhice aos 100 anos de idade: percepções na fala dos idosos. Estud. Interdiscipl. Envelhec.,; v. 19, n. 2, p. 583-598, 2014. 
Id on Line Revista Multidisciplinar e de Psicologia

Id on Line Multidisciplinary Journal and Psycology

BRAZ, I. A.; ZAIA, J. E.; BITTAR, C. M. L. Percepção da qualidade de vida de idosas participantes e não participantes de um grupo de convivência da terceira idade de Catanduva (SP). Estud. Interdiscipl. Envelhec.,; v. 20, n. 2, p. 583-596, 2015.

CARRETA, M. B.; BETTINELLI, L. A.; ERDMANN, A. L. Reflexões sobre o cuidado de enfermagem e a autonomia do ser humano na condição de idoso hospitalizado. Rev Bras Enferm.,; v. 64, n. 5, p. 958-62, 2011.

CHIN, Y. R.; LEE, I. S.; LEE, H. Y. Effects of hypertension, Diabetes, and/or Cardiovascular Disease on Health-related Quality of Life in Elderly Korean Individuals: A Population-based Cross-sectional Survey. Asian Nursing Research, v. 8, p. 267-273, 2014.

DAHMER, L.; DE OLIVEIRA, T. B.; KEMPER, C.; SANT’ANA, A. P.; DE MELO, G. L.; DE AVILA, J. G. Avaliação da qualidade de vida de pacientes hipertensos e diabéticos. Revista Contexto \& Saúde, Ijuí, v. 15, n. 28, p. 41-49, 2015.

DAWALIBI, N. W.; ANACLETO, G. M. C.; WITTER, C.; GOULART, R. M. M.; AQUINO, R. C. de. Envelhecimento e qualidade de vida: análise da produção científica da SciELO. Estudos de Psicologia, Campinas, v. 30, n. 3, p. 393-403, 2013.

FARIAS, R. G.; SANTOS, S. M. A. Influência dos determinantes do envelhecimento ativo entre idosos mais idosos. Texto Contexto Enferm, Florianópolis, v. 21, n. 1, p. 167-76, 2012. FOLSTEIN, M. F.; FOLSTEIN, S. E.; MCHUGH, P. R. Mini-mental state: a practical method for grading the cognitive estate of patients for the clinician. J Psychiatric Res., v. 12, p. 189$98,1975$.

FREITAS, M. C.; QUEIROZ, T. A.; SOUSA, J. A. V. O significado da velhice e da experiência de envelhecer para os idosos. Rev Esc Enferm, USP, v. 44, n. 2, p. 407-12, 2010.

GIL, A. C. Como elaborar projetos de pesquisa. 4. ed. São Paulo: Atlas, 2010.

GUEDES, D. V.; BARBOSA, A. J. G.; MAGALHÃES, N. C. de. Qualidade de vida de idosos com declínio cognitivo: auto e heterorrelatos. Aval. Psicol., v. 12, n. 1, p. 09-17, 2013.

LEITE, M. T.; WINCK, M. T.; HILDEBRANDT, L. M.; KIRCHNER, R. M.; DA SILVA, R. A. A. Qualidade de vida e nível cognitivo de pessoas idosas participantes de grupos de convivência. Rev. Bras. Geriatr. Gerontol., v. 15, n. 3, p. 481-492, 2012.

MACHADO, R. M. L.; CAVALIÉRE, S. L. O envelhecimento e seus reflexos biopsicossociais. Cadernos Unisuam, Rio de Janeiro, v. 2, n. 1, p. 110-120, 2012.

MALTA, D. C.; MOURA, L. de; PRADO, R. R. do; ESCALANTE, J. C.; SCHMIDT, M. I.; DUNCAN, B. B. Mortalidade por doenças crônicas não transmissíveis no Brasil e suas regiões, 2000 a 2011. Epidemiol. Serv. Saúde, v. 23, n. 4, p. 599-608, 2014.

NORONHA, D. D.; MARTINS, A. M. E. B. L. de; DIAS, D. S. dos; SILVEIRA, M. F.; DE PAULA, A. M. B.; HAIKAL, D. S. A. Qualidade de vida relacionada à saúde entre adultos e 
Id on Line Revista Multidisciplinar e de Psicologia

Id on Line Multidisciplinary Journal and Psycology

fatores associados: um estudo de base populacional. Ciência \& Saúde Coletiva, v. 21, n. 2, p. 463-474, 2016.

ORGANIZAÇÃO MUNDIAL DE SAÚDE (OMS). Whoqol-Old Instruções. Porto Alegre, RS: 2012.

PRAZERES, F.; FIGUEIREDO, D. Measuring quality of life of old type 2 diabetic patients in primary care in Portugal: a cross-sectional study. Journal of Diabetes \& Metabolic Disorders, v. 13, p. 68, 2014.

SANTOS, E.A. dos, TAVARES, D. M. S.; RODRIGUES, L. R.; DIAS, F. A.; FERREIRA, P. C. S. dos. Morbidades e qualidade de vida de idosos com diabetes mellitus residentes nas zonas rural e urbana. Rev Esc Enferm, USP, v. 47, n. 2, p. 393-400, 2013.

SANTOS, N. Qualidade de vida em mulheres mastectomizadas. Artigo (Enfermagem). FAINOR, 2015.

SILVA, L. M.; SILVA, A. O.; TURA, L. F. R.; MOREIRA, M. A. S. P.; RIDRIGUES, R. A. P.; MARQUES, M. C. Representações sociais sobre qualidade de vida para idosos. Rev Gaúcha Enferm., Porto Alegre, RS, v. 33, n. 1, p. 109-15, 2012.

STIVAL, M. M. de; LIMA, L. R.; FUNGHETTO, S. S.; SILVA, A. O.; PINHO, D. L. M.; KARNIKOWSK, M. G. O. Fatores associados à qualidade de vida de idosos que frequentam uma unidade de saúde do Distrito Federal. Rev. Bras. Geriatr. Gerontol., v. 17, n. 2, p. 395$405,2014$.

TAVARES, D. M. S.; GUIMARÃES, M. O.; FERREIRA, P. C. S.; DIAS, F. A.; MARTINS, N. P. F.; RODRIGUES, L. R. Quality of life and accession to the pharmacological treatment among elderly hypertensive. Rev Bras Enferm, v. 68, n. 6, p. 122-9, 2015.

TAVARES, D. M. S.; MARTINS, N. P. F.; DIAS, F. A.; DINIZ, M. A. Qualidade de vida de idosos com e sem hipertensão arterial. Rev. Eletr. Enf., v. 13, n. 2, p. 211-8, 2011. http://dx.doi.org/10.5216/ree.v13i2.10876.

WHO. World Health Organization. The World Health Organization quality of life assessment (WHOQOL): position paper from the World Health Organization. The WHOQOL Group 1995. Social Science and Medicine 10:1403-1409.

\section{Como citar este artigo (Formato ABNT):}

SOUZA, D.P.; MELO, T.S.; REIE, L.A.; LIMA, P.V. Qualidade de Vida em Idosos Portadores de Hipertensão Arterial e Diabetes mellitus. Id on Line Revista Multidisciplinar e de Psicologia, Out-Nov. de 2016, vol.10, n.31, Supl 3, p. 56-68. ISSN 1981-1179. 